\title{
Annie and FixIt: Showcasing Dynamic Guidance for Task-Based Exploratory Learning
}

\author{
James M. Thomas and R. Michael Young \\ Digital Games Research Center \\ Department of Computer Science \\ North Carolina State University, Raleigh, NC USA \\ jmthoma5@ncsu.edu, young@csc.ncsu.edu
}

The ITS field has benefitted from a shared consensus of proven techniques for intelligent scaffolding [4], but common techniques to solve the unique challenges of exploratory or inquiry-based tutoring have proven more elusive $[3,1]$.

Exploratory environments provide students with freedom to choose different courses of action. This complicates the tutor's ability to know what the student it trying to do, which introduces uncertainty in knowing whether or not a student has a misconception about the domain. When the tutor decides a misconception exists, it is difficult to know when is the right time to provide support to remediate that misconception, as the student may have changed focus to a different task. As others have noted [3], it is difficult to balance guidance with student exploration and "in such a way that learning is supported effectively, but the inquiry process is not reduced to following cookbook instructions."

Our system addresses these problems by leveraging a well-understood computational model of actions and the causal relationships between them used in automated planning. We have previously published the details of the design for this system [2], and recently completed the first full experimental evaluation of the system, which is being submitted to AIED 2011 as a full conference paper.

\section{References}

1. Quintana, C., Reiser, B., Davis, E., Krajcik, J., Fretz, E., Duncan, R., Kyza, E., Edelson, D., Soloway, E.: A Scaffolding Design Framework for Software to Support Science Inquiry. The Journal of the Learning Sciences 13(3), 337-386 (2004)

2. Thomas, J., Young, R.: Using Task-Based Modeling to Generate Scaffolding in NarrativeGuided Exploratory Learning Environments. In: Proceedings of the 14th International Conference on Artificial Intelligence in Education (2009)

3. Van Joolingen, W., De Jong, T., Dimitrakopoulou, A.: Issues in computer supported inquiry learning in science. Journal of Computer Assisted Learning 23(2), 111-119 (2007)

4. VanLehn, K.: The Behavior of Tutoring Systems. International Journal of Artificial Intelligence in Education 16(3), 227-265 (2006) 